\title{
Study of Dynamics SPAD and NDVI Values of Potato Plants According to the Differentiated Fertilization
}

\author{
Nina BĂRĂSCU ${ }^{1,2}$, Marcel M. DUDA ${ }^{1}$, Gheorghe OLTEANU ${ }^{2}$ \\ ${ }^{1}$ University of Agricultural Sciences and Veterinary Medicine Cluj-Napoca \\ ${ }^{2}$ National Institute of Research and Development for Potato and Sugar Beet \\ *corresponding author, e-mail: nina.barascu@potato.ro
}

Bulletin UASVM series Agriculture 73(1)/2016

Print ISSN 1843-5246; Electronic ISSN 1843-5386

DOI 10.15835/buasvmcn-agr: 12003

\begin{abstract}
Potato fertilization (especially nitrogen fertilization) has repercussions on the dynamics of plant growth and the production and quality. Recent results of research on the dynamics of NDVI and SPAD values of potatoes indicates ample opportunities to use these non-invasive measures to monitor the condition of vegetation in different crop growing conditions (Puiu et al. 2012, Shukla et al. 2007). The researches were conducted to obtain information on the possibilities of non-invasive assessment of NPK fertilization effects on the state of potato plants growing in nonirrigated conditions. Between 2013-2014, at NIRDPSB Brasov (Romania) were performed measurements of vegetation index NDVI, chlorophyll content and measurements of plant varieties Christian and Roclas harvested in dynamic, on two levels of nitrogen fertilization, N100 and N200 NPK reports 1: 1: 1 and 1: 0.9: 2. On the second decade of June 2013, the NDVI mean of Christian variety plants was 88.7, compared to 86.9 at Roclas variety. The SPAD values were 46.6 and 44.7. On 2014, a year with higher humidity, NDVI average values were higher on both varieties (91.6 and 90.8) and the SPAD values were lower (41.8 and 41.5). NDVI values of potato plants from fertilized variants were statistically differentiated according by variety, especially in the second part of the growing season. Through SPAD values differentiation of fertilization variants has been possible throughout the entire period observed. Hydrothermal conditions of vegetations limits the separation of fertilization variants with NDVI measurements, while SPAD values reflect supply plants with nitrogen.
\end{abstract}

Keywords: dynamics, NPK, NDVI, potato, SPAD

\section{INTRODUCTION}

Potato fertilization (especially nitrogen fertilization) has repercussions on the dynamics of plant growth and the production and quality. NPK fertilization effects on plant vegetation status can be monitored using non-invasive technologies. The most commonly used non invasive method is based on measurements with a chlorophyll meter device that can relate to leaf chlorophyll concentration (Goffart et al. 2008)

Recent results of research on the dynamics of NDVI (Normalized Difference Vegetation Index) and SPAD (Soil Plant Analysis Development) values of potatoes indicates ample opportunities to use these non-invasive measures to monitor the condition of vegetation in different crop growing conditions (Puiu et al. 2012, Shukla et al. 2007). The SPAD readings provide a better, rapid and convenient field method to monitor and regulate the $\mathrm{N}$ status of potato crop through crop growth period (Singh et al., 2007).

Assessment of crop nitrogen status throughout the growing season is indispensable in order to measure to optimize dynamic $\mathrm{N}$ supply (Shukla et al. 2007).

Measurements with the SPAD 502 meter produce relative SPAD meter values that are proportional to the amount of chlorophyll present in the leaf (Qihua et al., 2011 Puiu cited). The chlorophyll meter is an appropriate tool to 
determine the nutritional status in potato crops (Giltto et al., 2010). Chlorophyll contents in vegetation depend on soil nitrogen availability and on crop nitrogen uptake (Jongschaap et al., 2004).

Víga et al., 2012, showed that the SPAD values change at every foliar level and the measurements carried out on the middle foliar level provide the closest correlation with average SPAD values of the leaf canopy. For that, they recommend making measurements at different foliar levels or on the middle foliar level to determine the average SPAD value of the potato leaf canopy. The most important limitation is that factors other than available $\mathrm{N}$ (variety, growing season, location) can affect plant growth and chlorophyll development and thus the SPAD values obtained (Minotti et al., 1994).

Using SPAD 520 chlorophyll meter for monitoring the chlorophyll content of leave it may be noticed the existence of a possible shortage of nitrogen, early enough to be corrected in time without diminishing the production (SHAPIRO, 2006).

The NDVI handheld sensor can be used to follow crop growth and development throughout the season (Verhulst and Govaerts, 2010). The Field Scout CM 1000 measures reflectance in the red and near-infrared regions and estimates plant chlorophyll concentration (Davenport et al., 2005).

\section{MATERIALS AND METHODS}

Between 2013-2014, at Braşov, NDVI and SPAD measurements were performed and of plants harvested on dynamic for two mid-early potato varieties Christian and Roclas, on two levels of nitrogen fertilization $\mathrm{N} 100$ and $\mathrm{N} 200$ on reports NPK 1:1:1 and 1:0.9:2.

In the two years of study, it was aimed the dynamics of potato plant growth and tuber yield accumulation. It have been carried out 5-6 harvesting in dynamic for each variant of fertilization, since the 25 th day post emergence (2013) and the 22 nd day post emergence (2014). At each harvest moment were measured two plants/variant/replication. For each plant harvested were determined: plant height, number of stems/plant, the length of median leaf, the weight of aerial part, underground party, number of tubers/plant/, weight of tubers/plant.

At the same time were made on dynamic, non-invasive measurements, for determination of chlorophyll concentration from leaf using hand- held Chlorophyll Meter SPAD 502 created by Konica Minolta, Japan and measurements of vegetation index NDVI (Normalized Difference Vegetation Indices) using the apparatus FIELDSCOUT CM 1000 NDVI Meter at the wavelength of $660 \mathrm{~nm}$ and $840 \mathrm{~nm}$.

The values determined with the hand-held device SPAD 502 indicates the relative amount of chlorophyll present in the leaves, measured by the leaf transmittance on two wavelengths, $650 \mathrm{~nm}$ (red) and 940 (near infrared- NIR) (GIANQUINTO, 2004; Puiu, 2014).

Using portable devices SPAD 502 and CM 1000 Meter NDVI have made three measurements per plant/variant/replication. Determinations were performed on three plants/variation/repetition. Measurements were made on the 4th or 5th leaf from the tip of the plant down.

The research was conducted in two years with very different growth conditions in terms of climate. During the observations, were followed decadal average temperatures and precipitation amount.

In 2013 vegetation period was warm, with average temperature of $16,0^{\circ} \mathrm{C}$ and richer in precipitation (422.2 $\mathrm{mm}$ ), compared to the year 2014, at which the average air temperature, was 15. $0.3^{\circ} \mathrm{C}$ and there has been rainfall of $505.1 \mathrm{~mm}$

Hydrothermal Selianinov indices, calculate by reporting the amount of rainfall to the amount of monthly temperatures over $10^{\circ} \mathrm{C}$ (Seeman et al., 1979), for vegetation months, characterize 2013 as a dry year for potato (Elzbiata Radzka et al., 2015), the heavy drought in July has as result the shortening of the vegetation period, limiting tubers growth.

In 2014, in which the vegetation months were with richest rainfall and lower temperatures, the hydrothermal conditions were close to optimum levels during April-July, ensuring high yields in both varieties.

The research was conducted in two years with very different growth conditions in terms of climate. During the observations, were followed decadal average temperatures and precipitation amount.

In 2013 vegetation period was warm, with average temperature of $16,0^{\circ} \mathrm{C}$ and richer in precipitation (422.2 $\mathrm{mm}$ ), compared to the year 2014 , at which the average air temperature, was 15. $0.3^{\circ} \mathrm{C}$ and there has been rainfall of $505.1 \mathrm{~mm}$ 
In 2014 , in which the vegetation months were with richest rainfall and lower temperatures, the hydrothermal conditions were close to optimum levels during April-July, ensuring high yields in both varieties.

\section{RESULTS AND DISCUSSION}

Climatic conditions during vegetation period in the years 2013 and 2014 and have put a strong footprint on the growth and development of potato plants from Christian and Roclas similarly in both varieties.

In the two years studied, the average values of the plant measurements, SPAD and NDVI, for Roclas and Christian varieties at different times during vegetation period, are presented in tables 2 and 3. Analyzing comparatively the two data tables it is found that in 2014, compared to 2013, due to more favourable conditions for growth, plants have formed a richer foliage, the number of main stems and plant height being higher and a larger number of tubers was formed. Stronger growth of tubers has been supported by better foliage developed in favorable year.

Research on dynamic for SPAD and NDVI values on potato plants foliage were performed in order to specify the timing of potato plant growth, in which these measurements can bring significant information regarding the condition of the vegetation and the level of plant nitrogen supply in the various variants types of fertilizer.

In the year 2013 (tab. 4.) the first SPAD measurements carried out on June 20, have shown an average of 46.6 units for Christian variety and 44.7 units for Roclas variety. At subsequent measurements have been recorded progressive declines at Christian variety that were statistically significant starting with July 16.

On average, SPAD values were significantly influenced by nitrogen supply, average influence of NPK reports being insignificant.
On Christian variety, the highest SPAD value (47.9) was added in variant fertilized with N200 $\mathrm{kg} / \mathrm{ha}$, NPK ratio 1:2: $09 \mathrm{pm}$ on June 20 , then progressively decrease up to 37.9 on July 25 . Significantly lower were these values (44.1 and 23.9) in variant $\mathrm{N} 100 \mathrm{~kg} / \mathrm{ha}$ with the same NPK ratio. Potato plants of Roclas variety from variant N200, NPK ratio 1:9: 2 have reached SPAD values of 49.3 on July 16 , after which the decrease was only up to 48.1 in final measurement. Roclas variety at a nitrogen dose of N100 kg with an identical NPK ratio, had a SPAD value of 44.0 which declined to 26.1.

On both varieties, the variants with different nitrogen dose could be statistically separated by SPAD values throughout the entire period of observation to NPK ratio 1:0.9:2. Separation of nitrogen levels to NPK ratio 1:1: 1 was possible to all submissions only to Roclas variety, while to Christian variety only after July 16 . In the year 2014 (table 5.) the maximum SPAD values level at both varieties were measured on June 10 (on average 45.5 for Christian and 44.9 for Roclas).

From that date until the end of the measurements both varieties have registered significant declines of SPAD values continuously from one measurement to another, arriving on July, 21, at values 27.7 for Christian variety and 31.0 for Roclas. Significant declines have continued up to 23.0 and 21.6 on August, 5.

In climatic context of 2014, statistical separation of SPAD values between variants with N200 versus N100 regardless of NPK ratio was achieved during the period from June, 23 to August 5.

Due to the senescence advancement of the plants on measurements made on July 25, NDVI average went down heavily to both varieties, with values 60.4 - Christian and 64.2 - Roclas.

On Christian variety the effects of fertilization reports on NDVI values are insignificant until July 16, when, on the NPK 1:0.9:2 ratio, value (89.4)

Tab. 1. Average hydrothermal conditions during potato growing season (Braşov, 2013-2014)

\begin{tabular}{|c|c|c|c|c|c|c|c|c|}
\hline & Year & April & May & June & July & August & September & Vegetation period \\
\hline \multirow{2}{*}{ Average temperature ${ }^{\circ} \mathrm{C}$} & 2013 & 10.6 & 15.8 & 17.8 & 19.3 & 20.2 & 12.4 & 16.0 \\
\hline & 2014 & 9.1 & 13.9 & 16.4 & 19.3 & 18.7 & 14.3 & 15.3 \\
\hline \multirow{2}{*}{ Rainfall amount mm } & 2013 & 36.0 & 107.4 & 98.8 & 31.0 & 76.2 & 72.8 & 422.2 \\
\hline & 2014 & 118.5 & 100.2 & 76.0 & 115.4 & 60.6 & 34.4 & 505.1 \\
\hline
\end{tabular}


significantly exceeds the average value on the 1:1: 1 (86.6) and then decreases significantly at 56.7 compared with 64.2 due to loss of foliage. On Roclas variety significant decrease of NDVI values were found beginning with submissions from July 1.
On average the dose of $200 \mathrm{~kg}$ nitrogen/ ha compared with N100, resulted in significant declines of NDVI on potato plants from Christian variety on the first observations (June, 19 and July, 1). On Roclas variety have found significant increases in all measurements starting with July 1.

Tab. 2. The mean measurements of plant, SPAD și NDVI carried out in dynamics- 2013 la Braşov

\begin{tabular}{|c|c|c|c|c|c|c|c|c|c|}
\hline \multirow{2}{*}{ Measurement } & \multirow{2}{*}{ U.M. } & \multicolumn{2}{|c|}{19 June } & \multicolumn{2}{|c|}{1 July } & \multicolumn{2}{|c|}{ 16July } & \multicolumn{2}{|c|}{25 July } \\
\hline & & Christian & Roclas & Christian & Roclas & Christian & Roclas & Christian & Roclas \\
\hline Main stems & $\mathrm{nr} /$ plant & $4.3 \pm 1.5$ & $4.8 \pm 1.8$ & $4.2 \pm 1.6$ & $4.9 \pm 14$ & $3.7 \pm 1.3$ & $4.4 \pm 1.8$ & $3.8 \pm 1.1$ & $4.7 \pm 1.2$ \\
\hline Plant height & $\mathrm{cm}$ & $74 \pm 7$ & $71 \pm 6$ & $86 \pm 6$ & $83 \pm 7$ & $85 \pm 8$ & $86 \pm 7$ & $84 \pm 10$ & $86 \pm 10$ \\
\hline $\begin{array}{c}\text { Median } \\
\text { lenght leaf }\end{array}$ & $\mathrm{cm}$ & $36 \pm 3$ & $35 \pm 5$ & $34 \pm 3$ & $35 \pm 4$ & $32 \pm 2$ & $31 \pm 3$ & $12 \pm 14$ & $23 \pm 6$ \\
\hline $\begin{array}{c}\text { Number of } \\
\text { tubers }\end{array}$ & $\mathrm{nr} /$ plant & $13 \pm 6$ & $12 \pm 5$ & $13 \pm 5$ & $12 \pm 5$ & $14 \pm 5$ & $12 \pm 4$ & $14 \pm 3$ & 14 v 4 \\
\hline $\begin{array}{c}\text { Aerial part } \\
\text { weight }\end{array}$ & g / plant & $675 \pm 183$ & $666 \pm 231$ & $780 \pm 222$ & $885 \pm 231$ & $548 \pm 235$ & $643 \pm 198$ & $235 \pm 199$ & $343 \pm 200$ \\
\hline $\begin{array}{l}\text { Underground } \\
\text { part weight }\end{array}$ & g / plant & $66 \pm 20$ & $56 \pm 15$ & $68 \pm 23$ & $70 \pm 17$ & $57 \pm 20$ & $61 \pm 17$ & $21 \pm 17$ & $45 \pm 78$ \\
\hline $\begin{array}{l}\text { Tubers } \\
\text { weight }\end{array}$ & g / plant & $151 \pm 102$ & $127 \pm 113$ & $484 \pm 203$ & $453 \pm 249$ & $793 \pm 300$ & $646 \pm 201$ & $827 \pm 280$ & $767 \pm 264$ \\
\hline $\begin{array}{c}\text { Total biomas } \\
\text { weight }\end{array}$ & g / plant & $892 \pm 279$ & $849 \pm 288$ & $1333 \pm 374$ & $1407 \pm 388$ & $1399 \pm 507$ & $1350 \pm 344$ & $1083 \pm 400$ & $1154 \pm 335$ \\
\hline SPAD values & & $46.6 \pm 3.6$ & $41.7 \pm 3.4$ & $46.5 \pm 4.7$ & $45.6 \pm 2.9$ & $42.7 \pm 6.9$ & $41.7 \pm 6.0$ & $31.5 \pm 1.0$ & $37.6 \pm 7.0$ \\
\hline NDVI values & & $88.7 \pm 4.9$ & $86.2 \pm 4.4$ & $86.2 \pm 5.3$ & $85.6 \pm 5.2$ & $88.0 \pm 5.5$ & $85.4 \pm 5.3$ & $60.4 \pm 17.3$ & $62.9 \pm 9.3$ \\
\hline
\end{tabular}

Tab. 3. The mean measurements of plant, SPAD şi NDVI carried out in dynamics - 2014 la Braşov

\begin{tabular}{|c|c|c|c|c|c|c|c|c|c|c|c|}
\hline \multirow{2}{*}{ Measurement } & \multirow{2}{*}{ U.M. } & \multicolumn{2}{|c|}{10 June } & \multicolumn{2}{|c|}{23 June } & \multicolumn{2}{|c|}{7 July } & \multicolumn{2}{|c|}{21 July } & \multicolumn{2}{|c|}{5 August } \\
\hline & & Christian & Roclas & Christian & Roclas & Christian & Roclas & Christian & Roclas & Christian & Roclas \\
\hline Main stems & $\mathrm{nr} /$ plant & $8.2 \pm 1.9$ & $8.2 \pm 2.4$ & $8.5 \pm 2.1$ & $7.6 \pm 2.3$ & $7.7 \pm 1.6$ & $8.0 \pm 2.4$ & $6.0 \pm 2.0$ & $6.4 \pm 2.2$ & $0.6 \pm 1.7$ & $2.9 \pm 3.5$ \\
\hline Plant height & $\mathrm{cm}$ & $74 \pm 4$ & $76 \pm 5$ & $85 \pm 8$ & $89 \pm 11$ & $89 \pm 7$ & $94 \pm 9$ & $83 \pm 9$ & $93 \pm 12$ & $10 \pm 27$ & $41 \pm 48$ \\
\hline $\begin{array}{l}\text { Median } \\
\text { lenght leaf }\end{array}$ & $\mathrm{cm}$ & $31 \pm 3$ & $38 \pm 3$ & $27 \pm 3$ & $31 \pm 4$ & $31 \pm 3$ & $31 \pm 4$ & $13 \pm 16$ & $33 \pm 8$ & 0 & 0 \\
\hline $\begin{array}{l}\text { Number of } \\
\text { tubers }\end{array}$ & $\mathrm{nr} /$ plant & $19 \pm 5$ & $19 \pm 7$ & $19 \pm 5$ & $17 \pm 5$ & $18 \pm 3$ & $19 \pm 5$ & $16 \pm 4$ & $18 \pm 5$ & $18 \pm 3$ & $19 \pm 5$ \\
\hline $\begin{array}{l}\text { Aerial part } \\
\text { weight }\end{array}$ & g / plant & $807 \pm 227$ & $908 \pm 267$ & $895 \pm 284$ & $905 \pm 283$ & $578 \pm 128$ & $761 \pm 214$ & $229 \pm 119$ & $436 \pm 148$ & $13 \pm 39$ & $93 \pm 120$ \\
\hline $\begin{array}{l}\text { Underground } \\
\text { part weight }\end{array}$ & g / plant & $81 \pm 18$ & $82 \pm 19$ & $91 \pm 23$ & $89 \pm 30$ & $84 \pm 19$ & $86 \pm 23$ & $43 \pm 20$ & $65 \pm 27$ & $1 \pm 4$ & $10 \pm 12$ \\
\hline $\begin{array}{l}\text { Tubers } \\
\text { weight }\end{array}$ & g / plant & $201 \pm 79$ & $169 \pm 88$ & $490 \pm 122$ & $514 \pm 143$ & $759 \pm 172$ & $792 \pm 182$ & $808 \pm 194$ & $1010 \pm 327$ & $909 \pm 216$ & $1065 \pm 245$ \\
\hline $\begin{array}{l}\text { Total biomas } \\
\text { weight }\end{array}$ & g / plant & $1089 \pm 275$ & $1159 \pm 312$ & $476 \pm 361$ & $1508 \pm 383$ & $1420 \pm 286$ & $1639 \pm 384$ & $1078 \pm 304$ & $1510 \pm 474$ & $924 \pm 223$ & $1167 \pm 312$ \\
\hline SPAD values & & $45.2 \pm 3.5$ & $46.5 \pm 3.4$ & $41.6 \pm 3.1$ & $40.0 \pm 2.7$ & $35.8 \pm 4.4$ & $34.6 \pm 2.2$ & $29.5 \pm 6.7$ & $26.9 \pm 4.2$ & $22.3 \pm 10.8$ & $20.5 \pm 6.2$ \\
\hline NDVI values & & $90.2 \pm 3.9$ & $89.8 \pm 4.2$ & $91.2 \pm 2.7$ & $91.7 \pm 2.4$ & $87.2 \pm 5.0$ & $85.9 \pm 3.9$ & $81.4 \pm 6.6$ & $81.1 \pm 3.5$ & $63.2 \pm 25.6$ & $65.3 \pm 11.0$ \\
\hline
\end{tabular}


Systematic differences in NDVI values of the plants, due to different combinations of fertilization, were found at Roclas variety on July 16 and for Christian variety on July, 25.
Favorable climatic conditions for potato on the first part of vegetation (2014) are reflected in the NDVI values, in average 91.6 for Christian variety and 90.8 for Roclas - June, 23 (table 7).

Tab. 4. The effect of NPK fertilization on SPAD values during the vegetation of potato plants in Brasov in 2013

\begin{tabular}{|c|c|c|c|c|c|c|c|c|c|}
\hline \multirow{3}{*}{$\begin{array}{l}\text { Ratio } \\
\text { NPK }\end{array}$} & \multirow{3}{*}{$\begin{array}{c}\text { Dozes N } \\
\mathrm{Kg} / \mathrm{ha}\end{array}$} & \multicolumn{8}{|c|}{ SPAD values } \\
\hline & & \multicolumn{2}{|c|}{20 June } & \multicolumn{2}{|c|}{1 July } & \multicolumn{2}{|c|}{16 July } & \multicolumn{2}{|c|}{25 July } \\
\hline & & Christian & Roclas & Christian & Roclas & Christian & Roclas & Christian & Roclas \\
\hline \multirow{2}{*}{$1: 1: 1$} & 100 & $47.1 \mathrm{a}$ & $41.7 \mathrm{c}$ & $48.7 \mathrm{a}$ & $45.6 \mathrm{~b}$ & $39.2 \mathrm{~b}$ & $41.7 \mathrm{~b}$ & $28.6 \mathrm{~b}$ & $37.6 \mathrm{~b}$ \\
\hline & 200 & $47.2 \mathrm{a}$ & $46.2 \mathrm{a}$ & $46.7 \mathrm{a}$ & $47.4 \mathrm{a}$ & $45.8 \mathrm{a}$ & $49.9 \mathrm{a}$ & $35.6 \mathrm{a}$ & $45.8 \mathrm{a}$ \\
\hline \multirow{2}{*}{$1: 0,9: 2$} & 100 & $44.1 \mathrm{~b}$ & $44.0 \mathrm{~b}$ & $43.8 \mathrm{~b}$ & $42.1 \mathrm{c}$ & $40.5 \mathrm{~b}$ & $38.4 \mathrm{c}$ & $23.9 \mathrm{c}$ & $26.1 \mathrm{c}$ \\
\hline & 200 & $47.9 \mathrm{a}$ & $46.8 \mathrm{a}$ & $46.9 \mathrm{a}$ & $47.9 \mathrm{a}$ & $45.1 \mathrm{a}$ & $49.3 \mathrm{a}$ & $37.9 \mathrm{a}$ & $48.1 \mathrm{a}$ \\
\hline \multicolumn{10}{|c|}{ Means } \\
\hline \multicolumn{2}{|c|}{ Ratio $1: 1: 1$} & 47.1 & 44.0 & 47.7 & 46.5 & 42.5 & 45.8 & 32.1 & 41.7 \\
\hline \multicolumn{2}{|c|}{ Ratio 1:0,9:2 } & $46.0 \mathrm{~ns}$ & $45.4^{*}$ & $45.4^{\circ}$ & $45.0^{\circ}$ & $42.8 \mathrm{~ns}$ & $43.8 \mathrm{~ns}$ & $30.9 \mathrm{~ns}$ & 37.1 \\
\hline \multicolumn{2}{|c|}{ N $100 \mathrm{~kg} / \mathrm{ha}$} & 45.6 & 42.8 & 46.3 & 43.9 & 39.9 & 40.0 & 26.2 & 31.9 \\
\hline \multicolumn{2}{|c|}{ N $200 \mathrm{~kg} / \mathrm{ha}$} & $47.5 *$ & $46.5 *$ & $46.8 \mathrm{~ns}$ & $47.7 *$ & $45.5^{*}$ & $49.6 *$ & $36.8 *$ & $47.0 *$ \\
\hline \multicolumn{2}{|c|}{ Mean } & 46.6 & 44.7 & 46.5 & 45.8 & 42.7 & 44.8 & 31.5 & 39.4 \\
\hline \multicolumn{2}{|c|}{ Standard deviation } & 3.6 & 4.1 & 4.7 & 4.0 & 6.9 & 7.1 & 11.0 & 11.4 \\
\hline \multicolumn{2}{|c|}{$\mathrm{DL}_{\text {(RaportNPK) }} 5 \%$} & 1.2 & 1.3 & 1.5 & 1.3 & 2.3 & 2.4 & 3.6 & 3.7 \\
\hline \multicolumn{2}{|c|}{$\mathrm{DL}_{\mathrm{(N)}} 5 \%$} & 1.1 & 1.2 & 1.6 & 1.2 & 2.1 & 1.8 & 3.2 & 2.8 \\
\hline
\end{tabular}

Tab. 5. The effect of NPK fertilization on SPAD values during the vegetation of potato plants in Brasov in 2014

\begin{tabular}{|c|c|c|c|c|c|c|c|c|c|c|c|}
\hline \multirow{3}{*}{$\begin{array}{l}\text { Ratio } \\
\text { NPK }\end{array}$} & \multirow{3}{*}{$\begin{array}{c}\text { Dozes N } \\
\mathrm{Kg} / \mathrm{ha}\end{array}$} & \multicolumn{10}{|c|}{ SPAD values } \\
\hline & & \multicolumn{2}{|c|}{10 June } & \multicolumn{2}{|c|}{23 June } & \multicolumn{2}{|c|}{7 July } & \multicolumn{2}{|c|}{21 July } & \multicolumn{2}{|c|}{5 August } \\
\hline & & Christian & Roclas & Christian & Roclas & Christian & Roclas & Christian & Roclas & Christian & Roclas \\
\hline \multicolumn{12}{|c|}{ Interaction NPK ratio $* \mathrm{~N}$ doses } \\
\hline \multirow{2}{*}{ 1:1:1 } & 100 & $45.5 \mathrm{ab}$ & $46.5 \mathrm{a}$ & $41.0 \mathrm{~b}$ & $40.0 \mathrm{~b}$ & $32.8 \mathrm{c}$ & $34.6 \mathrm{~b}$ & $25.7 \mathrm{~b}$ & $26.9 \mathrm{~b}$ & $20.2 \mathrm{c}$ & $20.5 \mathrm{~b}$ \\
\hline & 200 & $46.8 \mathrm{a}$ & $45.7 \mathrm{a}$ & $43.4 \mathrm{a}$ & $43.3 \mathrm{a}$ & $39.7 \mathrm{a}$ & $38.7 \mathrm{a}$ & $27.0 \mathrm{~b}$ & $35.6 \mathrm{a}$ & $14.5 \mathrm{~d}$ & $30.2 \mathrm{a}$ \\
\hline \multirow{2}{*}{$1: 0,9: 2$} & 100 & $44.1 \mathrm{~b}$ & $43.2 \mathrm{~b}$ & $40.1 \mathrm{~b}$ & $39.6 \mathrm{~b}$ & $32.4 \mathrm{c}$ & $33.6 \mathrm{~b}$ & $27.1 \mathrm{~b}$ & $27.8 \mathrm{~b}$ & $26.0 \mathrm{~b}$ & $14.0 \mathrm{c}$ \\
\hline & 200 & $45.6 \mathrm{ab}$ & $44.2 \mathrm{~b}$ & $42.7 \mathrm{a}$ & $43.1 \mathrm{a}$ & $36.6 \mathrm{~b}$ & $37.9 \mathrm{a}$ & $31.1 \mathrm{a}$ & $35.2 \mathrm{a}$ & $31.3 \mathrm{a}$ & $22.0 \mathrm{~b}$ \\
\hline \multicolumn{12}{|c|}{ Means } \\
\hline \multicolumn{2}{|c|}{ Ratio 1:1:1 } & 46.1 & 46.1 & 42.2 & 41.7 & 36.3 & 36.6 & 26.4 & 31.2 & 17.4 & 25.3 \\
\hline \multicolumn{2}{|c|}{ Ratio 1:0,9:2 } & $44.8^{\circ}$ & $43.7^{\circ}$ & $41.4^{\circ}$ & $41.4^{\circ}$ & $34.5^{\circ}$ & $35.7^{\circ}$ & $29.1 *$ & $31.5 \mathrm{~ns}$ & $28.6^{*}$ & $18.0^{\circ}$ \\
\hline \multicolumn{2}{|c|}{ N $100 \mathrm{~kg} / \mathrm{ha}$} & 44.8 & 44.8 & 40.5 & 39.8 & 32.6 & 34.1 & 26.4 & 27.3 & 23.1 & 17.3 \\
\hline \multicolumn{2}{|c|}{ N $200 \mathrm{~kg} / \mathrm{ha}$} & $46.2 *$ & $44.9 \mathrm{~ns}$ & $43.0^{*}$ & $43.2^{*}$ & $38.2 *$ & $38.3^{*}$ & $29.0 *$ & $35.4^{*}$ & $22.9 \mathrm{~ns}$ & $26.1^{*}$ \\
\hline \multicolumn{2}{|c|}{ Mean } & 45.5 & 44.9 & 41.8 & 41.5 & 35.4 & 36.2 & 27.7 & 31.4 & 23.0 & 21.6 \\
\hline \multicolumn{2}{|c|}{ Standard deviation } & 3.6 & 3.3 & 3.2 & 3.1 & 4.7 & 4.1 & 6.8 & 6.0 & 12.4 & 9.0 \\
\hline \multicolumn{2}{|c|}{$\mathrm{DL}_{\text {(RaportNPK) }} 5 \%$} & 1.2 & 1.0 & 1.1 & 1.0 & 1.5 & 1.4 & 2.2 & 2.0 & 3.6 & 2.7 \\
\hline \multicolumn{2}{|c|}{$\mathrm{DL}_{\mathrm{N}} 5 \%$} & 1.2 & 1.1 & 1.0 & 0.8 & 1.2 & 1.2 & 2.2 & 1.5 & 4.1 & 2.6 \\
\hline
\end{tabular}



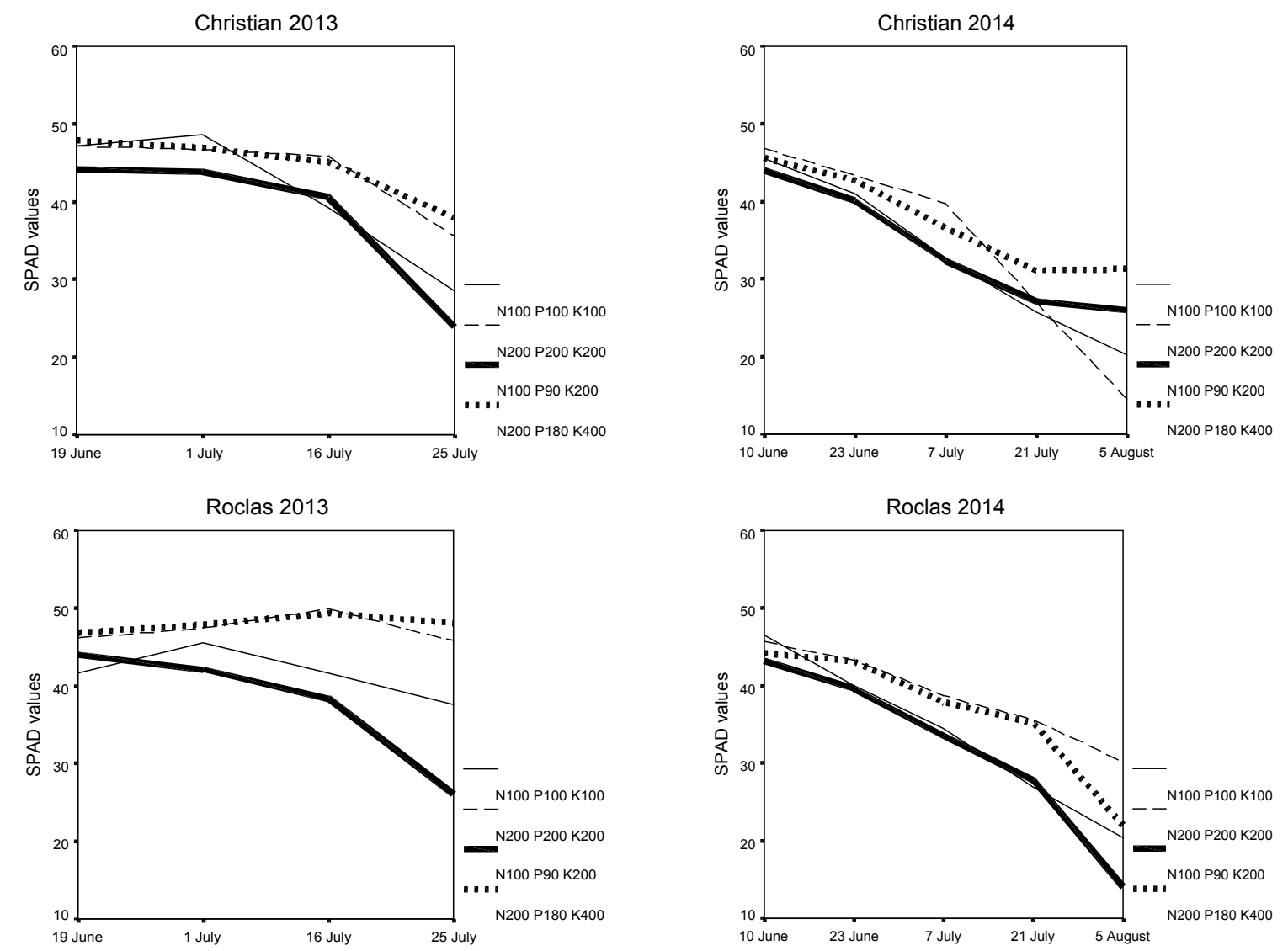

Fig. 1. Dynamics of average SPAD values - Christian and Roclas, Braşov 2013-2014

Tab. 6. The effect of NPK fertilization on NDVI values during the vegetation of potato plants - Brasov, 2013

\begin{tabular}{|c|c|c|c|c|c|c|c|c|c|}
\hline \multirow{3}{*}{$\begin{array}{l}\text { Ratio } \\
\text { NPK }\end{array}$} & \multirow{3}{*}{$\begin{array}{c}\text { Dozes N } \\
\mathrm{Kg} / \mathrm{ha}\end{array}$} & \multicolumn{8}{|c|}{ NDVI values } \\
\hline & & \multicolumn{2}{|c|}{19 June } & \multicolumn{2}{|c|}{1 July } & \multicolumn{2}{|c|}{16 July } & \multicolumn{2}{|c|}{25 July } \\
\hline & & Christian & Roclas & Christian & Roclas & Christian & Roclas & Christian & Roclas \\
\hline \multicolumn{10}{|c|}{ Interaction NPK ratio $* \mathrm{~N}$ doses } \\
\hline \multirow{2}{*}{$1: 1: 1$} & 100 & $90.4 \mathrm{a}$ & $86.2 \mathrm{a}$ & $86.8 \mathrm{a}$ & $85.6 \mathrm{a}$ & $87.3 \mathrm{~b}$ & $85.4 \mathrm{bc}$ & $69.6 \mathrm{a}$ & $62.9 \mathrm{~b}$ \\
\hline & 200 & $88.0 \mathrm{~b}$ & $86.6 \mathrm{a}$ & $84.9 \mathrm{a}$ & $86.1 \mathrm{a}$ & $85.9 \mathrm{~b}$ & $87.1 \mathrm{ab}$ & $58.8 \mathrm{~b}$ & $75.4 \mathrm{a}$ \\
\hline \multirow{2}{*}{$1: 0,9: 2$} & 100 & $88.7 \mathrm{ab}$ & $87.3 \mathrm{a}$ & $87.4 \mathrm{a}$ & $82.3 \mathrm{~b}$ & $88.3 \mathrm{ab}$ & $84.1 \mathrm{c}$ & $47.1 \mathrm{c}$ & $52.7 \mathrm{c}$ \\
\hline & 200 & $87.6 \mathrm{~b}$ & $87.4 \mathrm{a}$ & $85.7 \mathrm{a}$ & $85.2 \mathrm{a}$ & $90.5 \mathrm{a}$ & $87.8 \mathrm{a}$ & $66.3 \mathrm{a}$ & $65.8 \mathrm{~b}$ \\
\hline \multicolumn{10}{|c|}{ Means } \\
\hline \multicolumn{2}{|c|}{ Ratio 1:1:1 } & 89.2 & 86.4 & 85.9 & 85.9 & 86.6 & 86.3 & 64.2 & 69.1 \\
\hline \multicolumn{2}{|c|}{ Ratio 1:0,9:2 } & $88.1 \mathrm{~ns}$ & $87.4 \mathrm{~ns}$ & $86.6 \mathrm{~ns}$ & $83.8^{\circ}$ & $89.4 *$ & $85.9 \mathrm{~ns}$ & $56.7^{\circ}$ & $59.2^{\circ}$ \\
\hline \multicolumn{2}{|c|}{$\mathrm{N} 100 \mathrm{~kg} / \mathrm{ha}$} & 89.6 & 86.7 & 87.1 & 83.9 & 87.8 & 84.7 & 58.3 & 57.8 \\
\hline \multicolumn{2}{|c|}{ N $200 \mathrm{~kg} / \mathrm{ha}$} & $87.8^{\circ}$ & $87.0 \mathrm{~ns}$ & $85.3^{\circ}$ & $85.7^{*}$ & $88.2 \mathrm{~ns}$ & $87.4^{*}$ & $62.7 \mathrm{~ns}$ & $70.6^{*}$ \\
\hline \multicolumn{2}{|c|}{ Mean } & 88.7 & 86.9 & 86.2 & 84.8 & 88.0 & 86.1 & 60.4 & 64.2 \\
\hline \multicolumn{2}{|c|}{ Standard deviation } & 4.9 & 4.1 & 5.3 & 4.9 & 5.5 & 5.0 & 17.3 & 14.6 \\
\hline \multicolumn{2}{|c|}{$\mathrm{DL}_{\text {(RaportNPK) }} 5 \%$} & 1.6 & 1.3 & 1.8 & 1.6 & 1.8 & 1.6 & 5.6 & 4.6 \\
\hline \multicolumn{2}{|c|}{$\mathrm{DL}_{(\mathrm{N})} 5 \%$} & 1.6 & 1.3 & 1.7 & 1.6 & 1.8 & 1.6 & 5.7 & 4.3 \\
\hline
\end{tabular}


Tab. 7. The effect of NPK fertilization on NDVI values during the vegetation of potato plants - Brasov, 2014

\begin{tabular}{|c|c|c|c|c|c|c|c|c|c|c|c|}
\hline \multirow{3}{*}{$\begin{array}{l}\text { Ratio } \\
\text { NPK }\end{array}$} & \multirow{3}{*}{$\begin{array}{c}\text { Dozes N } \\
\mathrm{Kg} / \mathrm{ha}\end{array}$} & \multicolumn{10}{|c|}{ NDVI values } \\
\hline & & \multicolumn{2}{|c|}{10 June } & \multicolumn{2}{|c|}{23 June } & \multicolumn{2}{|c|}{7 July } & \multicolumn{2}{|c|}{21 July } & \multicolumn{2}{|c|}{5 August } \\
\hline & & Christian & Roclas & Christian & Roclas & Christian & Roclas & Christian & Roclas & Christian & Roclas \\
\hline \multicolumn{12}{|c|}{ Interaction NPK ratio $* \mathrm{~N}$ doses } \\
\hline \multirow{2}{*}{$1: 1: 1$} & 100 & $90.3 \mathrm{a}$ & $89.8 \mathrm{ab}$ & $91.8 \mathrm{a}$ & $91.7 \mathrm{a}$ & $88.4 \mathrm{a}$ & $85.9 \mathrm{~b}$ & $75.2 \mathrm{~b}$ & $81.1 \mathrm{c}$ & $54.4 \mathrm{~b}$ & $65.3 \mathrm{~b}$ \\
\hline & 200 & $91.4 \mathrm{a}$ & $89.9 \mathrm{ab}$ & $91.1 \mathrm{a}$ & $90.0 \mathrm{c}$ & $89.4 \mathrm{a}$ & $90.0 \mathrm{a}$ & $80.4 \mathrm{a}$ & $86.2 \mathrm{a}$ & $39.5 \mathrm{c}$ & $74.4 \mathrm{a}$ \\
\hline \multirow{2}{*}{$1: 0,8: 2$} & 100 & $90.4 \mathrm{a}$ & $88.3 \mathrm{~b}$ & $91.4 \mathrm{a}$ & $90.3 \mathrm{bc}$ & $83.8 \mathrm{~b}$ & $83.7 \mathrm{c}$ & $81.4 \mathrm{a}$ & $80.4 \mathrm{c}$ & $75.7 \mathrm{a}$ & $51.3 \mathrm{c}$ \\
\hline & 200 & $90.0 \mathrm{a}$ & $91.3 \mathrm{a}$ & $92.1 \mathrm{a}$ & $91.2 \mathrm{ab}$ & $87.7 \mathrm{a}$ & $88.5 \mathrm{a}$ & $82.0 \mathrm{a}$ & $84.5 \mathrm{~b}$ & $77.8 \mathrm{a}$ & $67.3 \mathrm{ab}$ \\
\hline \multicolumn{12}{|c|}{ Means } \\
\hline \multicolumn{2}{|c|}{ Ratio 1:1:1 } & 90.9 & 89.8 & 91.5 & 90.8 & 88.9 & 87.9 & 77.8 & 83.7 & 47.0 & 69.9 \\
\hline \multicolumn{2}{|c|}{ Ratio 1:0,9:2 } & $90.2^{\circ}$ & $89.8 \mathrm{~ns}$ & $91.8^{*}$ & $90.8 \mathrm{~ns}$ & $85.8^{\circ}$ & $86.1^{\circ}$ & $81.7^{*}$ & $82.5^{\circ}$ & $76.8 *$ & $59.3^{\circ}$ \\
\hline \multicolumn{2}{|c|}{$\mathrm{N} 100 \mathrm{~kg} / \mathrm{ha}$} & 90.3 & 89.0 & 91.6 & 91.0 & 86.1 & 84.8 & 78.3 & 80.8 & 65.1 & 58.3 \\
\hline \multicolumn{2}{|c|}{$\mathrm{N} 200 \mathrm{~kg} / \mathrm{ha}$} & $90.7 *$ & $90.6^{*}$ & $91.6 \mathrm{~ns}$ & $90.6^{\circ}$ & $88.6 *$ & $89.3 *$ & $81.2 *$ & $85.4^{*}$ & $58.7^{\circ}$ & $70.9 *$ \\
\hline \multicolumn{2}{|c|}{ Mean } & 90.5 & 89.8 & 91.6 & 90.8 & 87.3 & 87.0 & 79.8 & 83.1 & 61.9 & 64.6 \\
\hline \multicolumn{2}{|c|}{ Standard deviation } & 3.2 & 4.4 & 3.0 & 2.2 & 5.0 & 5.1 & 8.1 & 4.2 & 30.5 & 19.7 \\
\hline \multicolumn{2}{|c|}{$\mathrm{DL}_{(\text {Raport NPK })} 5 \%$} & 1.1 & 1.5 & 1.0 & 0.7 & 1.6 & 1.7 & 2.6 & 1.4 & 8.8 & 6.3 \\
\hline \multicolumn{2}{|c|}{$\mathrm{DL} 5 \%$} & 1.1 & 1.4 & 1.0 & 0.7 & 1.6 & 1.5 & 2.6 & 1.1 & 10.0 & 6.2 \\
\hline
\end{tabular}

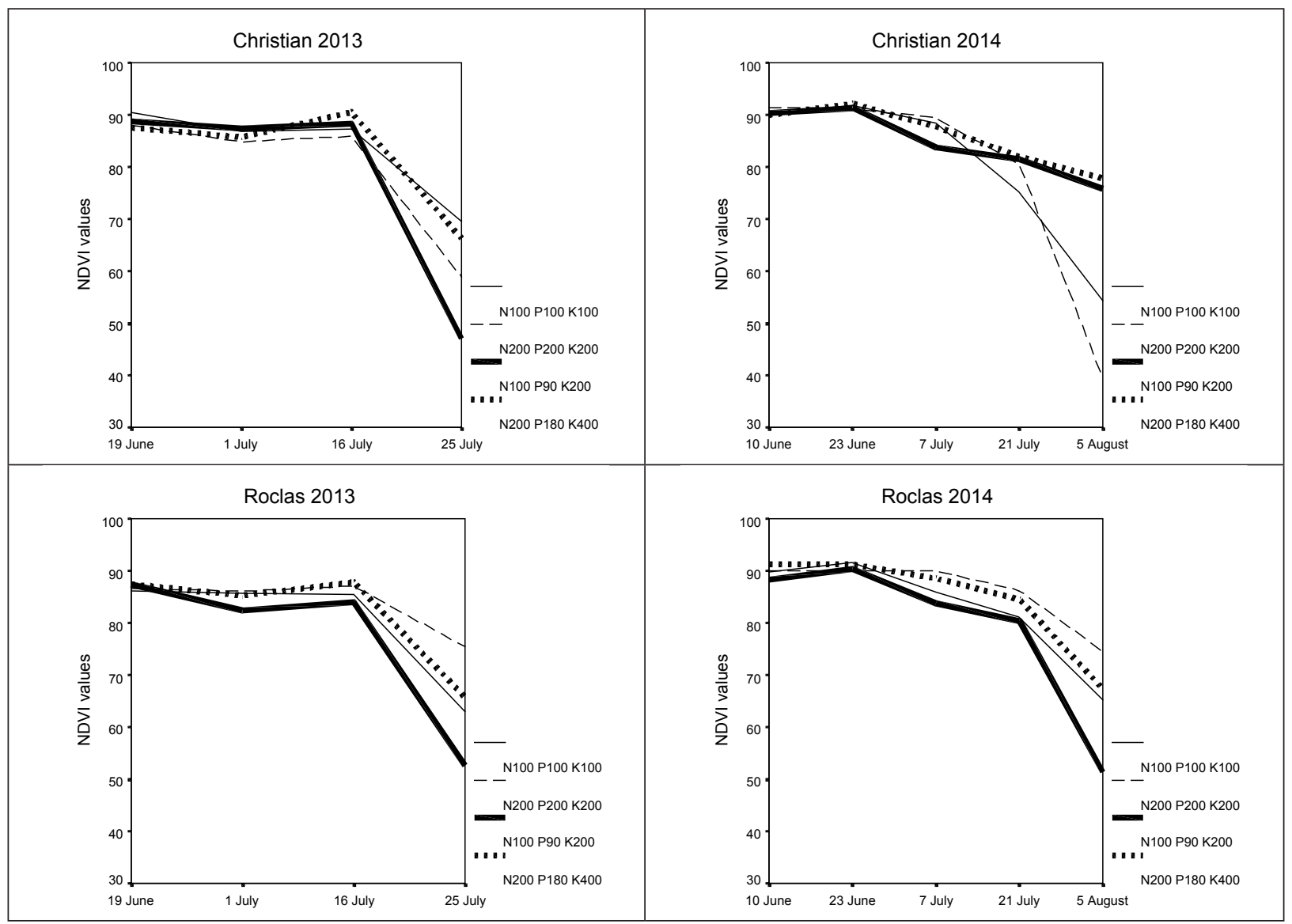

Fig. 2. Dynamics of average NDVI values - Christian and Roclas, Braşov 2013-2014 
On the observations made on June, 10 and June 23, depending on fertilization, NDVI values of Christian plants variety has not been differed significantly, while to Roclas variety is found to be slight differences, especially due to differentiation of nitrogen doses on NPK ratio 1:0.9:2.

In both varieties, beginning with observations from July, 7 until August 5 is found significant declines of NDVI values (61.9 - Christian and 64.6Roclas ).

On average, during this period the potato plants of both varieties from variants fertilized with $200 \mathrm{~kg} \mathrm{~N} /$ ha have a higher NDVI value comparatively with $100 \mathrm{~kg} \mathrm{~N} / \mathrm{ha}$ variant.

The NDVI value of the plant from fertilization variants were statistical distinguished on observations made in the period July, 7 to August, 5 for Christian variety and June 23 - August, 5 for Roclas variety.

The highest differences of NDVI values in both varieties were found to the observations made on August, 5 when the plants values of NDVI from fertilization variants have varied between 39.5 and 77.8 on Christian variety and 51.3 and 74.4 on Roclas variety.

Correlations between potato plants measurements and SPAD values for the years 2013 and 2014 are presented in table 8 . Comparing the correlation coefficients is found that in 2014 the most favourable climatic for potato the number of significant correlations is higher than in 2013. In both years the determined components of

Tab. 8. The correlation coefficients for dynamics of potato plants fertilization variants and dynamics SPAD values - Brasov, 2013 - 2014

\begin{tabular}{|c|c|c|c|c|c|}
\hline \multirow{3}{*}{ Measurement } & \multirow{3}{*}{ U.M. } & \multicolumn{4}{|c|}{ SPAD values } \\
\hline & & \multicolumn{2}{|c|}{2013} & \multicolumn{2}{|c|}{2014} \\
\hline & & Christian & Roclas & Christian & Roclas \\
\hline Main stems & $\mathrm{nr} /$ plant & 0.108 & 0.142 & $0.546^{* *}$ & $0.436^{* *}$ \\
\hline Plant height & $\mathrm{cm}$ & 0.205 & 0.194 & $-0.399 * *$ & -0.135 \\
\hline Median length leaf & $\mathrm{cm}$ & $0.686^{* *}$ & 0.203 & $0.263^{*}$ & $0.346^{* *}$ \\
\hline Number of tubers & $\mathrm{nr} /$ plant & -0.068 & $-0.266^{*}$ & $0.262^{*}$ & 0.061 \\
\hline Aerial part weight & g / plant & $0.805^{* *}$ & $0.565^{* *}$ & $0.724^{* *}$ & $0.752^{* *}$ \\
\hline $\begin{array}{c}\text { Underground part } \\
\text { weight }\end{array}$ & g / plant & $0.750^{* *}$ & $0.482^{* *}$ & $0.549^{* *}$ & $0.658^{* *}$ \\
\hline Tubers weight & g / plant & $-0.452^{* *}$ & $-0.407^{* *}$ & $-0.801^{* *}$ & $-0.682 * *$ \\
\hline Total biomass weight & g / plant & $0.252^{*}$ & 0.131 & $0.271^{*}$ & 0.198 \\
\hline
\end{tabular}

Tab. 9. The correlation coefficients for dynamics of potato plants fertilization variants and dynamics NDVI values - Brasov, 2013 - 2014

\begin{tabular}{|c|c|c|c|c|c|}
\hline \multirow{3}{*}{ Measurement } & \multirow{3}{*}{ U.M. } & \multicolumn{4}{|c|}{ NDVI values } \\
\hline & & \multicolumn{2}{|c|}{2013} & \multicolumn{2}{|c|}{2014} \\
\hline & & Christian & Roclas & Christian & Roclas \\
\hline Main stems & $\mathrm{nr} /$ plantă & 0.156 & 0.052 & $0.743^{* *}$ & $0.379^{* *}$ \\
\hline Plant height & $\mathrm{cm}$ & 0.007 & -0.63 & -0.210 & 0.006 \\
\hline Median length leaf & $\mathrm{cm}$ & $0.667^{* *}$ & $0.506^{* *}$ & $0.405^{* *}$ & 0.155 \\
\hline Number of tubers & nr / plantă (mv) & 0.029 & -0.214 & $0.226^{*}$ & 0.024 \\
\hline Aerial part weight & g / plantă (mv) & $0.728^{* *}$ & $0.694^{* *}$ & $0.728^{* *}$ & $0.732^{* *}$ \\
\hline $\begin{array}{c}\text { Underground part } \\
\text { weight }\end{array}$ & g / plantă (mv) & $0.761^{* *}$ & $0.254^{*}$ & $0.642^{* *}$ & $0.744^{* *}$ \\
\hline Tubers weight & g / plantă (mv) & $-0.369^{* *}$ & $0.516^{* *}$ & $-0.704^{* *}$ & $-0.585^{* *}$ \\
\hline Total biomass weight & g / plantă (mv) & $0.269^{*}$ & 0.11 & $0.419 * *$ & $0.311^{* *}$ \\
\hline
\end{tabular}


biomass harvesting in dynamics have correlated significantly with SPAD values measured on plants from variants with fertilizers.

In 2013, in both varieties, the number of main stems, plant height and number of tubers were not correlated with SPAD values. In favourable year 2014, the correlation of the number of tubers with SPAD values was significant for Christian variety.

Correlations between plant measurements and NDVI values for the years 2013 and 2014 are presented in table 9. In two years the correlation of plants measurements with NDVI values measured on potato plants side by side, from NPK variants, were similar with SPAD correlations values.

Correlations of biomass components with NDVI values of plants were statistically assured (p $=0.01 \%$ ) in both years and in both varieties.

The number of main stems was significantly correlated with NDVI values only in 2014 .

There was no correlation between plant height and NDVI values measured on the plants side by side. Correlations between NDVI and mid-leaf length were very tight for both varieties in 2013 and in 2014 for Christian variety only. in 2014 . In 2014 the correlation between the number of tubers and NDVI values was significant only for Christian variety.

\section{CONCLUSION}

On average, the SPAD values were significantly influenced by nitrogen supply, the influence of NPK reports being generally insignificant.

NDVI values of potato plants from fertilized variants were statistically differentiated according by variety, especially in the second part of the growing season.

Plant vegetation status indicated by the measurements of the NDVI was markedly different during the measurements in the years 2013 and 2014 on Roclas and Christian varieties.

In 2013, the maximum NDVI value of plant measured in the second decade of the month June was on average of 88.7 at Christian versus 86.9 at Roclas. In 2014, with higher precipitation, NDVI average values were higher in both varieties (91.6; 90.8).

At the beginning of vegetation, NDVI values measured on fertilizing plants variants are close and remain at high levels until mid-July in both years. Towards the end of the observation period, as a result of differentiated loss of plant foliage, NDVI values from variants with fertilization have statistically split depending on variety and fertilization variant.

At the end of the growing season, the highest NDVI values, have been recorded to Roclas, both in 2013 and 2014, on fertilized variant N200: P200: K200 (75.4 and 74.4).

On Christian variety the maximum NDVI values, have been recorded at different variants N100: P100: K100 (69.6) in 2013, and in 2014 the N200: P180: K400 (77.8).

SPAD measurements were distinct significantly correlated in both years with biomass components: aerial part weight, underground weight and weight of tubers..

The coefficients of correlation between biomass components and NDVI values were statistically assured $(\mathrm{p}=0.01 \%)$ in both years and in both varieties.

Through SPAD values differentiation of fertilization variants has been possible throughout the entire period observed. Hydrothermal conditions of vegetations limits the separation of fertilization variants with NDVI measurements, while SPAD values reflect supply plants with nitrogen.

Acknowledgements: This paper was published under the frame of European Social Fund, Human Resources Development Operational Programme 2007-2013, project no. POSDRU/159/1.5/S/132765.

\section{REFERENCES}

1. Davenport, J.R., E.M. Perry, N.S. Lang, R.G. Stevens, 2005, Leaf spectral reflectance for nondestructive measurement of plant nutrient status, Hortechnology, 15(1):31-35

2. Gianquinto, G., J.P. Goffart, M. Oliver, G. Guarda, M. Colauzzi, L. Dalla Costa, G. Delle Vedove, J. Vos, D.K.L. Mackerron, 2004, The use hand - held chlorophyll meters as tool to assess the nitrogen status and to guide nitrogen fertilization of potato crop, Potato Research 47, 35-80

3. Giletto Claudia Marcela, Cecilia Diaz, J. E. Rattin, H. E. Echeverria, D.O. Caldiz, 2010, Green index to estimate crop nitrogen status in potato processing varieties, Chilean Journal of Agricultural Research 70(1):142-149

4. Goffart, J.P., M. Olivier, M. Frankinet, 2008, Potato crop nitrogen status assessment to improve $\mathrm{N}$ fertilization management and efficiency: past-present-future, Potato Research, 51:355-383.

5. Jongschaap, R.E.E. And R. Booij, 2004, Spectral measurements at different spatial scales in potato: 
relating leaf, plant and canopy nitrogen status, International Journal of Applied Earth Observation and Geoinformation, vol. 5, Issue 3, 205-218.

6. Minotti, P.L., D.E. Halseth, J.B. Sieczka, 1994, Field chlorophyll measurements to assess the nitrogen status of potato varieties, HORTSCIENCE 29(12):1497-1500.

7. Puiu Isabela, G. Olteanu, G. Morar, Maria Ianoși, 2012, Monitoring the vegetation status of potato, Agricultura Ştiință și practică nr. 1-2(81-82).

8. Shukla, A. K., M.A. Khan, N.C. Upadhayay, B.P. Singh, S.S. Lal and S.K. Pandey, 2007, Chlorophyll meter: a tool for assessing dynamic nitrogen supply in potato crop under organic vis-a-vis inorganic nutrient management options, Potato Journal 34 (1-2):95-96.

9. Singh, J.P., R. Rachna, S.S. Lal, S.S. Ray, Sushma Panigrahy, 2007, Using leaf chlorophyll meter for N-fertilizer management in precision farming of potato, Potato Journal, 34(3-4): 221-226.

10. Shapiro, C.A., J.S. Schepers, D.D. Francis, J.F. Shanahan, 2006, Using a chlorophyll meter to improve $\mathrm{N}$ management, University of Nebraska- Lincoln Extension, Institute of Agriculture and Natural Resources, 13 - 16.

11. Verhulst, N., Govaerts, B.2010, The normalized difference vegetation index (NDVI) GreenSeekerTM handheld sensor: Toward the integrated evaluation of crop management. Part A: Concepts and case studies. Mexico, D.F.; CIMMYT.

12. Víga R, L. Huzsvaib, A. Dobosa, J. Nagy, 2012, Systematic measurement methods for the determination of the SPAD values of maize (Zea mays L.) canopy and potato (Solanum tuberosum L.), Communications in Soil Science and Plant Analysis, vol. 43, issue 12, 1648-1693. 